\title{
Review of: "Re-routing metabolism by the mitochondrial pyruvate carrier inhibitor MSDC-0160 attenuates neurodegeneration in a rat model of Parkinson's disease"
}

\author{
Heba Mansour ${ }^{1}$ \\ 1 Cairo University
}

Potential competing interests: The author(s) declared that no potential competing interests exist.

Thanks for sharing an interesting manuscript.

The manuscript is very interesting as it deals with the potential use of MSDC-0160 as metabolism modulator that inhibits mitochondrial pyruvate carrier (MPC). Accordingly, the cells rely more on other pathways to meet their energy needs, such as increasing glutamate oxidation, beta oxidation, and ketogenesis. Moreover, the authors stated that MSDC-0160 modulates autophagy and neuroinflammation. Thus, the topic chosen is of interest. However, there are some points to be addressed by the authors. Major points:

1-Figures of histopathological examination are needed for better clarification of results.

2- Inhibition of p-mTOR/mTOR is not sufficient to conclude that MSCD-0160 regulates autophagy. Other parameters must be evaluated to support this conclusion e.g. P62, LC3II/LC3I to enhance the novelty of the manuscript. Inhibition of p-mTOR/mTOR by MSCD-0160 has been reported by previous study [1].

3-The authors stated that MSDC-0160 has anti-inflammatory effect. Immunofluorescent images of some inflammatory markers may be beneficial to endorse this conclusion.

Minor points:

1- Unclear how many animals are analyzed in each figure.

2- Some extra figures based on the new experimental evidences can be added to address above concerns.

3- The entire bibliography should be reviewed since all references are represented as et al.

\section{References}

1. AAnamitra Ghosh, Trevor Tyson, Sonia George, Erin N. Hildebrandt, et al. (2016). Mitochondrial pyruvate carrier regulates autophagy, inflammation, and neurodegeneration in experimental models of Parkinson's disease. Sci. Transl. Med., vol. 8 (368). doi:10.1126/scitranslmed.aag2210. 
